
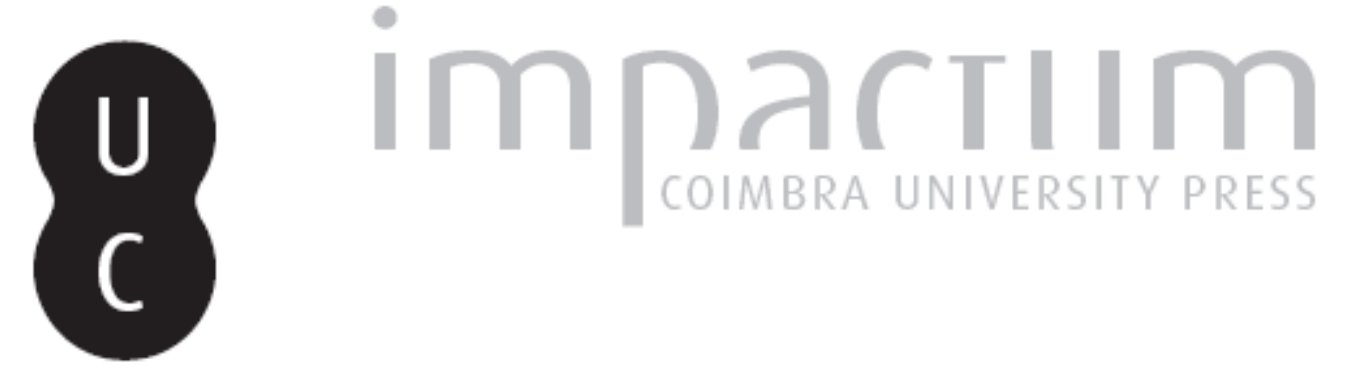

\title{
Le role de la partie intermediaire (Thumos) dans la tripartition de l'ame
}

\author{
Autor(es): Renaut, Olivier
}

Publicado por: Imprensa da Universidade de Coimbra

URL persistente:

URI:http://hdl.handle.net/10316.2/42224

DOI:

DOI:https://doi.org/10.14195/2183-4105_6_1

Accessed : $\quad$ 26-Apr-2023 08:50:42

A navegação consulta e descarregamento dos títulos inseridos nas Bibliotecas Digitais UC Digitalis, UC Pombalina e UC Impactum, pressupõem a aceitação plena e sem reservas dos Termos e Condições de Uso destas Bibliotecas Digitais, disponíveis em https://digitalis.uc.pt/pt-pt/termos.

Conforme exposto nos referidos Termos e Condições de Uso, o descarregamento de títulos de acesso restrito requer uma licença válida de autorização devendo o utilizador aceder ao(s) documento(s) a partir de um endereço de IP da instituição detentora da supramencionada licença.

Ao utilizador é apenas permitido o descarregamento para uso pessoal, pelo que o emprego do(s) título(s) descarregado(s) para outro fim, designadamente comercial, carece de autorização do respetivo autor ou editor da obra.

Na medida em que todas as obras da UC Digitalis se encontram protegidas pelo Código do Direito de Autor e Direitos Conexos e demais legislação aplicável, toda a cópia, parcial ou total, deste documento, nos casos em que é legalmente admitida, deverá conter ou fazer-se acompanhar por este aviso.

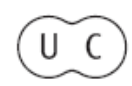




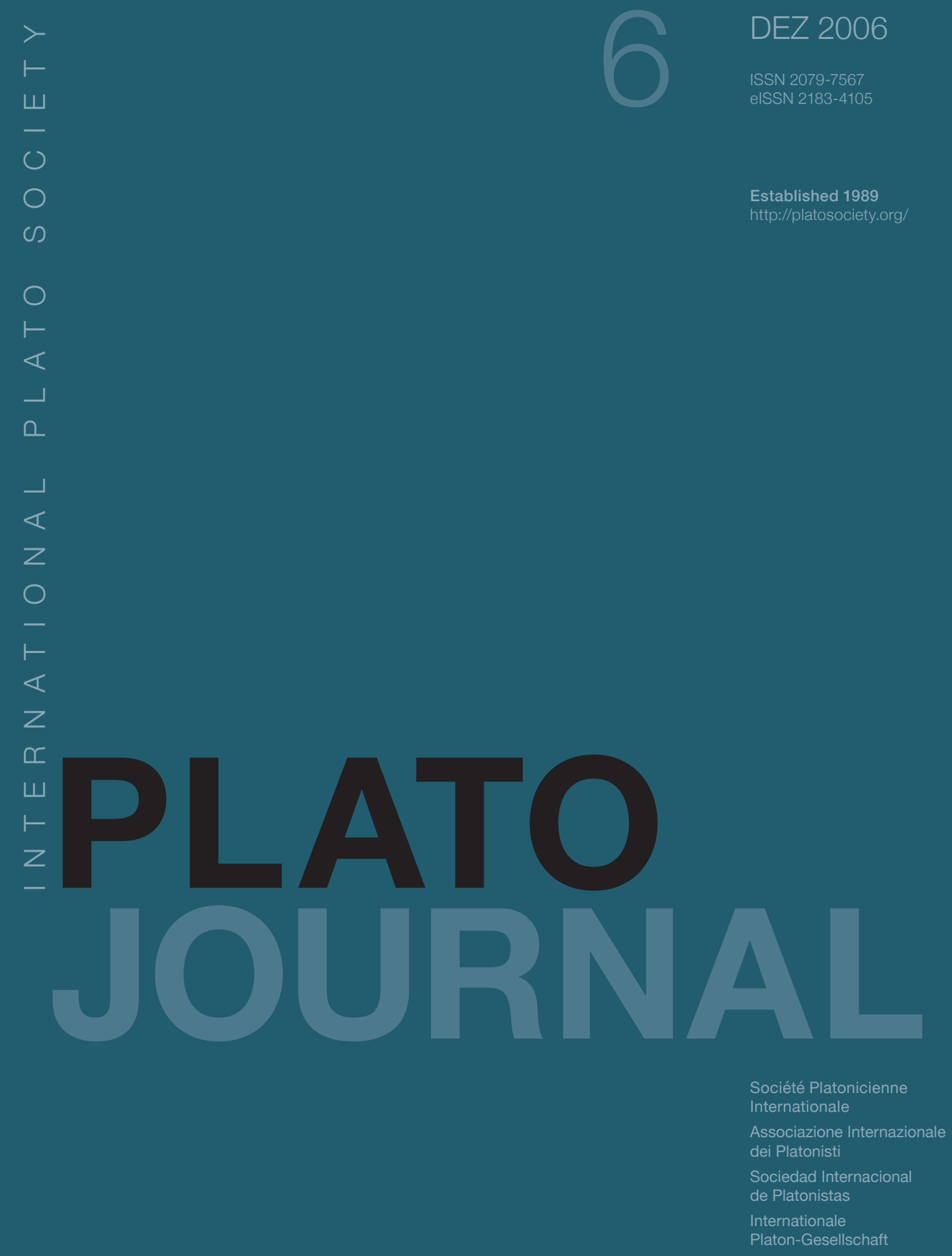




\section{LE ROLE DE LA PARTIE INTERMEDIAIRE (THUMOS) DANS LA TRIPARTITION DE L'AME}

Dans la République, Socrate décrit une âme en proie à une " dissension » (stasis) qui révèle sa structure tripartite. Au livre IX (580d7-581b10), Socrate s'emploie à qualifier trois caractères constitués par l'hégémonie d'une des parties de l'âme qui asservit les deux autres : celui dont la partie désirante commande est « ami du gain » (philokerdès), celui dont c'est la partie intermédiaire est « ami des honneurs et de la victoire » (philótimos et philónikos), enfin celui dont c'est la partie rationnelle est appelé « philosophe » (philósophos). Les trois fonctions de l'âme se définissent donc par un « désir » et un plaisir propres, dont l'objet est respectivement le plaisir (hédonē), l'honneur $($ timē), et la vérité ou la pensée. Socrate impute ainsi à chacune de ces parties un ensemble d'actions dont les motivations diffèrent. Plusieurs commentateurs ont examiné la consistance psychologique de ce qui pourrait apparaître comme une doctrine platonicienne ${ }^{1}$. Cependant, parce que la tripartition de l'âme est corrélative de la partition sociale en vertu de l'analogie entre l'âme et la cité, on ne peut isoler une analyse psychologique des actions et des affections de l'âme de sa fonction éthique et politique ; la tripartition de l'âme aurait pour vocation d'offrir un modèle structurel permettant de forger les caractères des citoyens, voire de les faire parvenir à la vertu ${ }^{2}$. La solidarité des dimensions psychologique d'une part et politique et éthique d'autre part est clairement affirmée selon nous à travers la découverte de la partie intermédiaire de l'âme, la partie « ardente » (thumós). Le thumós est un élément qui se singularise par son affinité avec une nature animale et brutale, mais qui peut être éduqué, dans la mesure où il est le siège d'émotions « politiques $»^{3}$. Nous proposons donc de relire la doctrine de la tripartition à partir d'une analyse du thumós ; ce biais permet en effet de montrer que le but de la tripartition de l'âme est de tracer les linéaments d'une anthropologie politique : diviser l'âme, c'est certes reconnaître une différence entre des

1 Moline, J., «Plato on the complexity of the psyche », Archiv fûr Geschichte der Philosophie, 1978, 1-26 ; Penner, T., "Thougt and desire in Plato", in G.Vlastos, (ed.), Plato II : Ethics, Politics, and the Philosophy of Art and Religion, Garden City, Anchor Books, 1971, 96-118 ; RoBINSON, R., "Plato's separation of reason from desire", Phronesis, 16, 1971, 38-48 ; WoOdS, M., "Plato's division of the soul", in Proceedings of the British Academy 73, 1987, p.23-48.

2 Voir les articles importants de COOPER, J., «Plato's theory of human motivation », History of Philosophy Quarterly, 1, 1984, 3-21 ; CORNFORD, F.M., « Psychology and social structure in the Republic», Classical Quarterly, 6, 1912, p.262-264. RIsT, J.M., "Plato says that we have tripartite souls. If he is right, what can we do about it? », in M.-O., Goulet-Cazé, Madec G., O’Brien, D., (ed.), Mélanges Jean Pépin, "Sophiès maiètores », "Chercheurs de Sagesse », Hommage à Jean Pépin, Institut d'Études Augustiniennes, Paris, 1992, 103-124. Voir également AnNAS, J., An Introduction to Plato's Republic, New-York, Clarendon Press, 1981, en particulier le chapitre V "Parts and virtues of state and soul", p.109sq.

${ }^{3}$ À propos de la fonction éthique et politique du thumós dans les dialogues de Platon, on peut consulter tout d'abord FrERE, J., Les Grecs et le désir de l'Etre, Paris, Les Belles-Lettres, 1981 et récemment Ardeur et Colère, Le thumós platonicien, Paris, Kimé, 2004 ; puis, pour une analyse plus détaillée HoBBS, A., Plato and the Hero, Cambridge, Cambridge University Press, 2000 ; et enfin les analyses importantes de Wersinger, A.-G., Platon et la dysharmonie, Paris, Vrin, 2001, en particulier les chapitres VII à IX. 
principes d'action d'inégales valeurs, mais c'est aussi cerner différents « moteurs » de l'action potentiellement vertueuse dans le cadre d'une communauté politique.

Nous exposerons d'abord brièvement comment au livre IV de la République le thumós se singularise des deux autres parties du fait de son intermédiarité, sans pour autant fragiliser l'unité de l'âme. Ensuite, nous montrerons que Socrate attribue au thumós une fonction importante dans l'exercice et l'acquisition de la vertu ; la reconnaissance du thumós comme fonction psychique à part entière correspond à un infléchissement d'une théorie intellectualiste de la vertu et à une revalorisation des émotions vertueuses. Enfin, nous évoquerons les éléments de continuité décelables dans le Phèdre et le Timée, dans la mesure où ces deux dialogues continuent d'élaborer les fondements théoriques d'une anthropologie de l'action humaine.

Comme dans une guerre civile, l'unité de l'âme est fragilisée par l'opposition de forces et de raisonnements qui sont les principes de l'action $(436 \mathrm{a} 8-\mathrm{b} 3)^{4}$. La tension intérieure de l'âme révèle, sinon des " parties » réellement distinctes ontologiquement, du moins une structure psychique, dont les pôles sont comme des fonctions d'un caractère donné ${ }^{5}$. Socrate invoque donc les cas de conflit intérieur de l'âme afin d'éprouver son unité : la guerre intestine, si elle est politiquement perçue comme le pire des maux de la cité, a le mérite en psychologie de révéler une structure composée d'éléments différenciés.

En 436b8-c1, Socrate recourt au principe de non-contradiction ${ }^{6}$ pour distinguer d'abord la partie appétitive de la partie rationnelle. C'est apparemment ce même principe auquel Socrate fait appel pour révéler l'existence de la partie intermédiaire : le thumós apparaît tout d'abord distinct de la partie appétitive (439e1-440e7), puis de la partie rationnelle (440e8-441c3). La tripartition de l'âme est donc issue d'un raisonnement qui démontre successivement qu'il existe une opposition possible entre

${ }^{4}$ On trouve un exposé complet sur les différentes lectures psychologiques du « conflit de l'âme » dans l'ouvrage de PRICE, A.W., Mental Conflict, London \& New-York, Routledge, 1995, ch. 1 et 2, p.8-103.

5 Au livre IV de la République, à partir de 435b, Socrate emploie le terme eîdos $(435 \mathrm{c} 1, \mathrm{c} 5)$ pour désigner ces parties de l'âme en écho au terme génos $(435 \mathrm{~b} 5, \mathrm{~b} 7)$ pour les groupements sociaux, bien que le vocabulaire ne soit pas figé. Nous comprenons qu' « êldos » est ici un équivalent de « structure », chacune de ces parties désignant une unité conceptuelle d'une multiplicité de mouvements de l'âme, ou d'actions ; ce terme ne semble ni fragiliser l'unité de l'âme, ni non plus désigner une « Forme » de l'âme, puisque la tripartition n'est encore qu'une hypothèse.

6 La valeur de ce principe fait débat. Certains interprètes préfèrent l'expression de « Principe des Opposés », puisque l'application de ce principe porte sur des contraires plus que sur des contradictoires. Voir à ce sujet ROBINSON, R.,art.cit.; et MiLLER, F.D. Jr, : « Plato on the Parts of the Soul », in VAN OPHUIJSEN, J.M., (ed.), Plato and Platonism, Studies in Philosophy and the History of Philosophy, vol. 33, The Catholic University of Amercia Press, Washington DC, 1999, p.84-101. 
deux des trois parties de l'âme ${ }^{7}$. Glaucon propose d'abord de réduire le thumós à l'epithumía (439e5), dans la mesure où ils sont tous deux des élans et non des entraves. Socrate lui oppose deux récits et leur interprétation afin de prouver que le thumós est une partie indépendante des deux autres. Le premier est l'histoire de Léontios (439e6440b7) qui, contre son désir morbide de regarder les cadavres oppose tout d'abord un mouvement de pudeur en se masquant le visage, puis, vaincu par son désir, adresse un blâme à ses propres yeux, et par métonymie à lui-même. La « colère » (orgê) , selon Socrate, peut donc se porter sur des désirs, ce qui apparaît suffisant pour distinguer la partie appétitive d'une autre partie, tout aussi impulsive, le thumós. Socrate invoque également l'exemple d'hommes justes dont on doit imputer l'endurance à cette partie de l'âme. Afin de prêter au thumós une autonomie relativement à la partie rationnelle, il faut cependant montrer que Léontios se masque le visage en vertu d'une disposition éthique non réfléchie, conceptuellement indépendante de la raison, et c'est la fonction du second récit. Le second récit (441b2-c2) est une référence homérique à Ulysse (Odyssée, XX, 17), que Socrate réinterprète pour distinguer la partie rationnelle capable de délibérer du thumós qui est naturellement impulsif et prompt à la vengeance. Ce n'est plus cette fois la colère qui adresse un blâme aux désirs, c'est la raison elle-même qui blâme la colère.

Dans cette démonstration, le thumós apparaît bien comme une partie « intermédiaire », et non pas tierce : il demeure en effet une ambiguïté concernant son autonomie. Tout d'abord, on peut remarquer que dans chacune de ces anecdotes, Socrate ne désigne jamais le thumós lui-même, mais ce qui lui est traditionnellement apparenté : dans l'histoire de Léontios, le thumós se manifeste par la colère (orgē), alors que dans la citation d'Homère, il est assimilé à l'organe du cœur (kradiē $)^{8}$. Ensuite, le thumós ne se distingue pas encore, à l'instar des autres parties, par un désir qui lui est propre. En effet, la partie appétitive et la partie rationnelle se différencient non seulement à travers l'opposition de leur force mais aussi par l'objet de leur désir ; ainsi, les parties appétitive et rationnelle constituent des pôles structurels de l'action qui recoupent la distinction entre l'âme et le corps ; la partie intermédiaire en revanche ne se définit dans l'un et l'autre exemple que par l'assistance qu'elle procure à l'une ou l'autre des parties, et ce de manière exclusive ${ }^{9}$. Et assurément, il est difficile d'imaginer

7 Comme nous l'a fait remarquer un lecteur anonyme de cet article, et qu'il soit remercié ici, Socrate analyse des états de tension (partie rationnelle-partie appétitive ; partie appétitive-thumós; thumós-partie rationnelle), et non des parties indépendantes les unes des autres, ce qui nous semble exclure à la fois l'hypothèse selon laquelle Socrate réifie ces parties à l'intérieur de l'âme, mais aussi l'hypothèse selon laquelle chacune des parties doit être comprise à la manière d'homoncules, comme le soutiennent par exemple J.ANNAS, op.cit, et plus récemment encore C. BOBONICH, Plato's Utopia Recast, Oxford, Clarendon Press, 2002.

8 Cette assimilation est loin d'être anodine, dans la mesure où chez Homère, le thumós désigne un fluide qui défie des catégories fixistes de l'âme et du corps. Sur cette question, on pourra consulter A. CHEYNS, «Recherche sur l'emploi des synonymes ètor, kèr, kradiè, dans l'Iliade et l'Odyssée », Revue Belge de Philologie et d'Histoire, 63 (1), 1985, p.15-73, et également Sullivan Darcus, S., Psychological and Ethical Ideas. What Early Greeks say. E.J.Brill, New-York-Köln, 1995.

9 Socrate évite ainsi l'objection qui fait du thumós le siège du dilemme, une âme déchirée, pouvant prêter main forte aux deux autres parties en même temps (440b4-7), comme c'est traditionnellement le cas chez Homère ou Euripide. Sur cette question, 
une situation où partie rationnelle et partie appétitive se ligueraient ensemble contre cette partie intermédiaire, ce qui pousse certains commentateurs à douter de la consistance psychologique et même logique du thumós ${ }^{10}$. On ne saurait en effet déterminer, du moins au livre IV, quel est l'objet de son désir puisqu'il est le siège d'émotions dont il n'est pas aisé de déterminer le genre. Et cependant, Socrate lui assure au moins lexicalement une certaine indépendance, en le nommant tò thumoeidès, comme pour lui conférer une autonomie fonctionnelle et conceptuelle ${ }^{11}$.

Une solution à ce manque relatif d'autonomie consiste à faire du thumós une partie strictement « énergique », la réduisant à une simple « force » capable d'assister l'une ou l'autre des deux autres parties. C'est ce à quoi nous invite la métaphore militaire, qui fait du thumós un simple allié, prenant les armes contre la partie appétitive (440e5-6). Il faudrait ainsi comprendre que le thumós est le centre de la « motivation » dans l'action, synthétisant ainsi les raisons qu'un individu a d'agir de telle manière et son désir d'agir d'une autre manière ${ }^{12}$.

Cette interprétation n'épuise cependant pas la fonction du thumós dans l'âme et dans la cité. Le thumós est une énergie qui recèle potentiellement une orientation éthique : c'est pourquoi Socrate déclare que « la plupart du temps » (440b6; 440e4-5), le thumós est davantage sensible aux recommandations de la raison, quoique sa nature puisse être corrompue (441a3). En effet, dans l'histoire de Léontios, le thumós semble sensible à une certaine norme qui lui interdit de considérer comme un spectacle ce qui est une réalité morbide ; de même Ulysse condamne sa colère, non pas tant parce qu'elle est injuste (au contraire, le sentiment de némesis est justifié au spectacle de l'infidélité de ses servantes), mais parce que son caractère intempestif ne sert pas sa vengeance calculée. Le thumós serait donc le siège d'émotions potentiellement vertueuses, que le discours permet d'orienter. Un bref détour sur la conception du thumós avant la République est nécessaire pour en cerner la puissance.

nous renvoyons principalement aux analyses de C. GILL, Personality in Greek Epic, Tragedy, and Philosophy: The Self in Dialogue. New York, Oxford University Press, 1996.

10 Pour cette position, voir PENNER, T., art.cit. Y. Bres, dans La Psychologie de Platon,Paris, P.U.F., 1968, p. 311-319, montre selon nous avec raison que le thumós constitue, sur le plan strictement psychologique, une partie dont la fonction est moins englobante qu'une théorie socratique érotique.

11 Nous nous accordons ainsi avec la manière dont C.SHIELDS comprend ces parties de l'âme dans son article : « Simple Souls », in WAGNER, E., (ed.), Essays on Plato's Psychology, Lexington Books, Lanham, 2001, p.137-156. Néanmoins, il semble que l'autonomie nominale du thumós est assurée avant d'être conceptuellement consistant, puisque ce n'est qu'au livre IX que Socrate lui attribue un objet de désir : la timè ; au livre IV, le thumós est seulement le siège de l'emportement, de l'endurance, et d'émotions plus subtiles comme la réserve et le respect.

12 Telle est l'interprétation qui se dégage par exemple de l'analyse de J.COOPER, art.cit. 
Déjà dans le Protagoras, Platon évoque la fonction psycho-politique de cet élément hérité d'Homère ${ }^{13}$. Le sophiste évoque le thumós dans deux passages radicalement distincts. "Thumós » est d'abord le terme utilisé en doublet avec kólasis et nouthétēsis pour désigner une « colère » indignée devant le spectacle d'une injustice (323c4-e3). Le thumós est le soutien d'un sentiment moral qui ne se manifeste que si l'individu manque de la vertu politique exigée, étant donnée qu'elle est le fruit non du hasard ou de la nécessité, mais bien de la «culture » ou de l'éducation. Plus loin dans le dialogue, en 351a-b, Protagoras évoque une fois encore le thumós mais dans le sens d'une « ardeur » qui entre comme composante de l'audace du courageux, contrairement à l'audacieux qui n'est pas courageux, et qui tient son audace d'une mauvaise nature, ou d'une mauvaise éducation. Dans les deux cas, le thumós est le siège d'émotions, violentes, mais en affinité avec le sentiment de l'excellence vertueuse (aretē) dont le courage (andreía) est le parangon. En d'autres termes, dans la bouche de Protagoras, le thumós semble bien être le soutien d'une éthique et d'une politique fondée sur l'émotion vertueuse, qu'il désigne dans son mythe sous le terme d'aidōs ${ }^{14}$. De la même manière, la question de l'éducation du thumós est présente en filigrane dans le Banquet. Dans son discours inaugural, bien qu'il condamne la recherche d'honneurs, Phèdre déclare que le sens de la honte et de l'honneur, traditionnellement attaché au thumós, est l'instrument de l'excellence (178d1-178e3). Le fait de participer au sens de la honte constitue selon lui le fondement même de l'éthique, et conditionne la participation de l'individu aux autres vertus, du courage notamment (178e3-179b3). Le caractère exemplaire, et tout autant édifiant, des sacrifices d'Alceste et d'Achille montre que l'émotion est potentiellement le moteur de l'action vertueuse.

Dans les deux cas, Socrate refuse au thumós la dimension éthique ou politique que ses interlocuteurs veulent lui donner. Dans le Protagoras, Socrate reprend dans son argumentation le terme "thumós » mais le considère comme une force qu'il met sur un pied d'égalité avec le plaisir, la peine, l'amour et la crainte, privant la science de sa dúnamis $(352 \mathrm{~b}-\mathrm{c})$. Socrate réduit donc le thumós à une passion, et ne reconnaît pas sa vocation éthique. De même dans le Banquet, le thumós d'Achille que Phèdre prenait

13 Pour un compte-rendu de la notion de thumós chez Homère, voir principalement la monographie de CHEYNS, A., «Le thumós et la conception de l'homme dans l'épopée homérique », Revue Belge de Philologie et d'Histoire, LXI, 1983, 20-86. Plus précisément, H. PELlicCiA, dans Mind, Body, and Speech in Homer and Pindar, Hypomnemata, 107, Vandenhoeck und Ruprecht, Göttingen, 1995, examine comment c'est le thumós qui engage l'individu dans une communauté de valeurs partagées grâce à un système discursif complexe. Cette étude nous invite donc à penser que le thumós est l'intermédiaire dialogique privilégié dans la relation politique. Pour les enjeux de sa réappropriation dans les dialogues de Platon, nous renvoyons à A.-G. WERSINGER, op.cit. qui en cerne les enjeux épistémologiques et éthiques; et à A.HoBBS, op.cit. dont l'étude concerne les dialogues socratiques jusqu'à la République et dont le centre est l'andreia.

${ }_{14}$ Pour un essai d'interprétation du fonctionnement de la loi et de la norme dans le mythe de Protagoras, nous renvoyons à BRISSON, L., « Le mythe de Protagoras. Essai d'analyse structurale », Quaderni Urbinati di Cultura Classica 20, 1975, p. 7-37 ; CAssin, B., L'Effet Sophistique, Paris, Gallimard, 1995 ; NARCY, M., « Le contrat social : d'un mythe moderne à l'ancienne sophistique ", Philosophie 28, 1990, p.32-56. 
comme exemple d'une éthique du don et de la générosité (179e-180b), n'est plus dans la bouche de Diotime, qu'un élan vers une image erronée de l'immortalité (208c-e).

Ces deux exemples montrent assez que dans la République au contraire, Socrate interroge la valeur éthique et politique d'une action guidée par une représentation qui ne suit pas un ordre rationnel. En d'autres termes, il s'agit de questionner le degré moral de l'émotion spontanée de l'individu, dont la vertu n'est pas à proprement parler une vertu, mais une disposition à la vertu. Les sentiments moraux comme le sens de la honte (aidôs) sont appelés à partir de la République à jouer un rôle non négligeable dans l'exercice de la vertu ${ }^{15}$. La tripartition de l'âme doit se substituer à la polarité raison-désir, afin de garantir à la fois l'hégémonie de la partie rationnelle, et également de lui adjoindre la puissance des émotions vertueuses.

Le thumós, soutien éthique et politique de la conduite héroïque chez Homère, doit dans la République véhiculer la puissance de la loi, et pour ce faire être réformé de telle manière que son agressivité ne se porte pas sur la raison, et ne puisse agir indépendamment d'elle. Cette stratégie, est à l'œuvre dans les livres II et III de la République. Le terme thumós y apparaît, indépendamment de la tripartition, comme un « naturel » plus qu'une " partie » de l'âme, c'est-à-dire un ensemble de dispositions, et qui fait pendant au «naturel philosophe » (375a-e). En 411a-e, Socrate se livre à un véritable catalogue des caractères dont la variété correspond précisément au degré de tension et de relâchement du thumós, par l'effet de la musique et de la gymnastique ${ }^{16}$. Certes la définition du « naturel philosophe » ne s'épuise pas dans une correspondance avec un certain degré de tension du thumós, mais c'est justement parce que cette « partie » ne se laisse pas intellectualiser que son opposition logique avec le logistikón est rendue nécessaire. Il faut donc édifier le thumós, l'éduquer de manière à ce que la loi soit intériorisée telle une norme ${ }^{17}$. Ainsi, on éduque le thumós en recourant à des discours, des mythes, autant de " modèles éducatifs », qui portent en eux la trace des formes de la vertu. Selon A.Hobbs, un modèle éducatif est nécessaire pour véhiculer de manière immanente les normes de justice afin qu'elles soient agies de manière spontanée $^{18}$. Le recours au modèle éducatif comme le sont les héros chez Homère, sert ainsi à produire un èthos et une héxis. Aussi la République met-elle en œuvre une subtile relecture du contenu de l'épopée, tout en conservant le mode mimétique comme moyen d'imprégnation de la vertu. Le produit en est une inversion de la valeur traditionnelle des héros : Achille est blâmé du fait de son thumós débridé (386c-391c), alors qu'Ulysse est pris deux fois en exemple (390d4-5 et 441b2-c2) pour engager l'âme au dialogue entre ses propres parties. Et cependant, c'est paradoxalement la

15 Cette hypothèse est évoquée à plusieurs reprises par C.KAHN, Plato and the Socratic Dialogues, Cambridge, Cambridge University Press, 1996, notamment p.137-142 à l'occasion de l'analyse du Gorgias.

${ }^{16}$ Ce catalogue est analysé par A.-G.Wersinger, op.cit., p.172-179, qui le compare ensuite à la typologie des caractères au livre IX de la République, en en montrant la série de glissements, p. 185-191.

17 Nous pourrions reprendre ici l'expression judicieuse de M.Narcy, art.cit. p.44 : « La

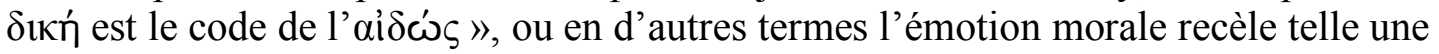
norme intériorisée ce que la loi édicte d'une manière transcendante. Seulement, il nous semble qu'il faille attribuer cette tentative à Socrate dans la République, plutôt qu'à Protagoras dans le dialogue éponyme.

18 A.Hobbs, op.cit. voir en particulier les ch.6 et 7. 
figure d'Achille, condamné dans le livre III pour l'húbris de son thumós, qui est mise en avant au livre VI, en 516d5-6, puisqu'il fait preuve d'une prothumía envers la fin éthique qu'il s'est lui-même proposée, pour rendre sensible les interlocuteurs de Socrate à l'enthousiasme du philosophe à l'endroit de la vérité.

L'attention portée à l'éducation de cette partie de l'âme pose nécessairement la question de la valeur éthique d'une action commise par ce même thumós. Or, la République présente bien deux champs lexicaux de la vertu, le premier est systématisé par les quatre vertus cardinales, et le second comprend un ensemble de vertus, ou de conduites vertueuses : respect, pudeur, amitié, sens de la honte. Ces « petites règles » (425a8-9) qui découlent spontanément d'une éducation systématique (424a4-b1), appellent précisément le thumós comme siège d'une impulsion vertueuse endocinétique. Deux modèles de l'action vertueuse se font donc écho sans qu'on puisse toutefois parvenir à les relier de manière satisfaisante : le second, qui fait de l'émotion un moteur potentiellement vertueux, prend le relais du premier, fondé sur le primat de la connaissance. Il nous semble que la question dans la République n'est pas de distinguer celui qui possède la vertu de celui qui ne la possède pas relativement à la connaissance qu'il en a, savoir ou opinion droite ${ }^{19}$; mais de comprendre comment dans une communauté politique, non seulement la cité, mais aussi les membres qui la composent accèdent à la vertu.

Le thumós est donc doublement intermédiaire : parce qu'il est sensible à la valeur que la communauté reconnait à un objet ou à une action, il est intermédiaire entre la raison et l'appétit, puisqu'il fait précéder la dimension axiologique d'un objet ou d'une action sur le plaisir qu'il peut procurer, tout en ne sachant pas nécessairement ce qui fonde sa valeur. Le thumós est l'instrument intermédiaire de l'exercice de la vertu, non pas certes une vertu intellectualisée, mais une vertu spontanée. C'est pourquoi il est intermédiaire en un autre sens : si c'est bien le thumós qui conserve une opinion droite concernant la vertu, alors, dans l'action, il pourrait bien être considéré par Platon comme le moyen le plus sûr de lier l'individu à la communauté politique, dans la mesure où chaque individu devient le miroir des valeurs de la cité, et la cité le garant de toutes les valeurs éthiques : le thumós est l'interface entre éthique individuelle et justice politique ; chacun, par son intermédiaire, devient le témoin de ses propres actions (X, 603c10-608d10).

La fonction du thumós comprise en ce sens éclaire nous semble-t-il le sens de l'analogie de l'âme et de la cité, puisque parallèlement à sa fonction épistémologique, elle apparaît clairement comme la justification d'une entreprise éducative qui s'adresse

19 C'est par exemple le parti de l'interprétation de C.BoBOnich, Plato's Utopia Recast, op.cit., qui oppose de manière radicale le Phédon et la République d'une part et les dialogues plus tardifs; les premiers nient selon lui la possibilité pour un individu de commettre réellement un acte vertueux par opinion droite, les seconds réalisant cette possibilité anthropologique, notamment en abandonnant une interprétation rigide de la tripartition de l'âme. Nous adoptons au contraire une position résolument unitariste : la République prépare la réforme de la sensibilité qui a cours dans les Lois en réévaluant justement la valeur dynamique des émotions comme la réserve, le sens de la honte, et la sensibilité à l'honneur. 
au thumós des individus ${ }^{20}$. En effet, on doit envisager le thumós de deux manières, selon l'ordre de préséance des motifs psychologique et politique. Tout d'abord, on peut considérer comme une donnée que le thumós entendu comme fonction psychologique, et donc indépendamment de chacune des classes envisagées, doit occuper une place intermédiaire au sens où il est le relais d'une raison législatrice ; par métaphore, le thumós doit se faire sentinelle de la raison. Mais politiquement, il faut que chacune des classes occupe « de bon gré » sa position, et c'est alors en diffusant, par l'intermédiaire du thumós, une opinion droite relative à la justice que la cité est harmonisée. Par ailleurs, dans le cas des auxiliaires, qui par métaphore sont le thumós de la cité, il faudra non seulement faire qu'ils soient justes, mais aussi courageux, en vertu de la prépondérance de leur thumós dans leur caractère ; et nous pourrions ajouter que sans un thumós éduqué, il est à craindre que la modération ne puisse être le propre de la classe des producteurs-artisans ; de même pour le philosophe, si son thumós ne secondait pas dans toutes ses entreprises la raison, on pourrait douter de sa vocation à gouverner. L'analogie ainsi entendue justifie selon nous l'entreprise éducative de la République et l'introduction du thumós dans la psychologie platonicienne la rend possible.

\section{- III -}

Il demeure cependant une difficulté concernant la puissance du thumós. S'il est bien un intermédiaire permettant, précisément à cause de sa résonance archaïque, de fluidifier l'exercice de la vertu, il risque également de fragiliser l'ordre architectonique entre les fonctions de l'âme, et partant, entre les classes sociales. C'est pour cette raison qu'au livre IX, Socrate précise que la partie intermédiaire n'est pas seulement un moteur de l'action, mais se singularise également par un type de désir : la timè, inférieure en valeur relativement à la vérité et à la connaissance. Cette seconde tripartition opère selon nous un renversement important : le thumós n'estime plus seulement un objet ou une action en fonction de la valeur que lui prête une communauté donnée, mais désire et prend plaisir à l'honneur en tant qu'objet ${ }^{21}$. Aussi,

20 Dans son article fondamental, «The analogy of City and Soul in Plato's Republic», in Lee, Mourelatos et Rotry (dir.), "Exegis and Argument », Phronesis, supp. 1, Assen, 1973, p. 196-206., B.WILLIAMS expose de manière convaincante comment l'analogie de l'âme et de la cité se heurte à des problèmes d'interprétations presque insolubles, puisque la relation tout-parties produit des contradictions concernant la théorie de la vertu, et que l'isomorphisme âme-cité ne rend pas compte de la nécessaire communication entre les deux. D'une manière tout aussi convaincante et dans une perspective psychanalytique cette fois, J. LEAR, dans « Inside and Outside the Republic », Phronesis, 37, 1992, p. 184-215, explique que l'analogie est l'expression de la communication entre l'individu et la cité, par le biais de la culture. Ces deux lectures ne sont pas incompatibles. Nous souscrivons aux conclusions des deux auteurs, en proposant l'hypothèse que l'analogie explique théoriquement comment les dirigeants peuvent de manière efficace, en s'appuyant sur le thumós des citoyens, diffuser une opinion droite relative à l'ordre de la cité.

21 Sur la notion fondamentale de timè, on consultera RiEDINGER, J.-C., « Remarque sur la $\tau \mu \mu$ chez Homère », Revue des Études Grecques, 1976, n89, p.244-264, ainsi que «Les deux aidôs chez Homère », Revue de Philologie, de Littérature et d'Histoire 
comme nous l'avons dit, face à la tendance du thumós à contaminer toutes les sphères de l'action, il convient de lui adresser également un discours qui lui rappelle qu'il n'est qu'un intermédiaire. En d'autres termes, si un état pacifique est souhaitable dans l'âme en harmonisant ses fonctions quitte à ce que l'action soit accomplie seulement en vertu d'une opinion droite, la dissension du thumós avec les deux autres parties doit demeurer présente à l'esprit de chacun afin de justifier l'hégémonie de la raison. C'est à ce réquisit que répondent les discours sur l'âme tripartite dans le Phèdre et dans le Timée, dont nous n'évoquerons ici que la dimension protreptique.

Le Phèdre revient sur la manière dont le thumós, parce qu'il est sensible aux discours, peut être exhorté afin que son attachement à la timè devienne un moteur suffisant pour engager l'âme dans un désir de connaissance, en se dépassant pour ainsi dire lui-même. La tripartition de l'âme ne persiste dans ce dialogue que sous la forme du mythe de l'attelage ailé, et où le nom des parties de l'âme ne nous est présenté que par indices, laissant entendre que l'idée de l'âme n'est pas accessible (246a3-6). L'allégorie d'origine homérique, jouant sur l'ambiguité de l'incarnation de l'âme, semble ainsi s'adresser directement au thumós de Phèdre (244e7-8); la valeur symbolique de l'image permet alors de produire un discours édifiant. Même les dieux qui dans la République se singularisent par la simplicité de leur âme sont dans le Phèdre des attelages ailés, ce qui présuppose dans l'âme du dieu l'achèvement d'une dynamique que doit imiter son cortège $\mathrm{e}^{22}$. On retrouve alors deux émotions dont le siège est le thumós chez Homère réinterprétées au sein d'une psychagogie érotique : le bon cheval ressent de l'aidôs (253d6 et 254e9), sentiment ambigu qui tout à la fois signale la persistance d'un désir et la force qui empêche son achèvement; tandis que le cocher ressent de la sébas, respect craintif du divin $(254 \mathrm{~b} 8)$. Si la tripartition dans le Phèdre ne poursuit pas un but clairement éducatif, elle permet de représenter l'âme à elle-même dans une dynamique érotique qui, loin de contredire la hiérarchie des parties de la République, la complète grâce à la rhétorique : l'âme, que l'incarnation force à diversifier ses fonctions, peut néanmoins s'unifier dans un mouvement qui redonne au corps sa juste place ; le thumós constitue un moteur pour la reconnaissance de l'hégémonie de la raison.

Le Timée à son tour établit une physiologie qui seconde la psychologie tripartite (69c2-70d6) : le thumós, parce qu'il est encore compris à travers une métaphore militaire et politique, aura ainsi pour fonction naturelle de «politiser» le corps. En effet, dans ce dialogue, la tripartition de l'âme est relatée au sein d'un eikos logos, un « discours vraisemblable ${ }^{23}$ explicitement relié à la République. Cependant, la

Anciennes, Tome LIV, Fas.1, 1980, p.62-80. Pour le rôle de cette notion dans les dialogues de Platon, voir LARRIVEe, A., « « Du souci à l'honneur de l'âme. Aspects de la timè dans les Lois de Platon », Kairos, 19, 2002, pp.111-127, bien que nous pensions, en accord avec A.-G. WERSINGER, op.cit. p.227-257, que cette notion est déjà largement analysée et prise en compte dès les premiers dialogues.

${ }^{22}$ Sur cette question, voir les remarques éclairantes de CHERLONNEIX, J.-L., « L'âme de Platon et ses deux corps », in Mélanges Jean Pépin, "Sophiès maiètores », "Chercheurs de Sagesse », Hommage à Jean Pépin, M.-O., Goulet-Caze, Madec G., O'BrIEn D., (ed.), Institut d'Études Augustiniennes, Paris, 1992, p.93-102.

23 Sur l'héritage pythagoricien et médical qui pourrait transparaître dans la présentation de la tripartition dans le Timée, voir J.FrERE, «Thumós et Kardia, Timée, 69c2-70d6 », Kléos, 1997, vol.1, n¹, p.1-8. 
perspective physiologique sur le thumós est tout à fait absente de la République. Pourquoi faut-il attribuer au thumós le soutien physiologique de la kardia ? L'âme tripartite dans le Timée ne l'est que parce qu'elle est incarnée : le thumós et la partie appétitive sont des parties mortelles qui périssent avec le corps ; mais le naturalisme éthique du Timée n'est pas contradictoire avec la fonction prescriptive de la représentation imagée d'une âme tripartite. Le thumós a justement un rôle intermédiaire entre l'âme incarnée et le corps, et c'est pourquoi le dérèglement moral est aussi le signe d'un dérèglement physiologique. Le thumós communique avec le cœur, mais, contrairement à la République, ils sont clairement distingués : le thumós est intermédiaire entre le corps organique et le corps signifiant, sur-déterminé par les métaphores de la frontière, de la sentinelle et de la conquête ${ }^{24}$. Le corps est décrit comme un territoire que l'âme, à défaut de conquérir tout à fait, arpente, quadrille et surtout occupe en signifiant sa présence ${ }^{25}$. Si le thumós semble être à première vue être réduit dans le Timée à l'émotion colérique, Platon lui prête dans ce dialogue une fonction éthique, dont le but est analogiquement comparé à une fin militaire et politique. Avec le thumós, Platon rend compte non seulement de la naturalité de l'âme en proie avec le corps, mais exige aussi de cette âme qu'elle veille à ne pas s'y asservir.

Le thumós est une force, non-rationnelle plutôt qu'irrationnelle, que l'éducation modèle afin d'obtenir un èthos ou une hèxis compatible avec une organisation politique juste. La tripartition de l'âme est donc bien une hypothèse, dont la vérité ne repose pas tant sur la réalité ontologique des parties dans l'âme, mais sur sa valeur à la fois explicative et protreptique. Dans la République, le thumós est un intermédiaire au sens où il est déjà conçu comme le véhicule d'émotions potentiellement vertueuses; le Phèdre et le Timée peuvent dès lors être compris comme une théorisation des effets d'une conception tripartite de l'âme : on doit s'adresser au thumós de l'individu pour lui signifier son ambivalence; le thumós a ainsi une double fonction : permettre à l'âme incarnée de reconnaître la supériorité de la raison et de la valeur de son désir, et rappeler à cette même âme qu'elle prime sur le corps qu'elle doit s'efforcer d'habiter de la manière la plus divine possible, en luttant contre lui. Les émotions vertueuses dont le thumós est le siège, si elles manquent de la conscience de leur propre valeur, n'en demeurent pas moins un fondement essentiel de l'éthique platonicienne, ainsi que de l'exercice de la philosophie.

\section{Olivier RENAUT}

Université Paris 1 Panthéon-Sorbonne.

\footnotetext{
${ }^{24}$ Voir les remarques de PENDER, E., «The language of the Soul in the Timaeus », in Interpreting the Timaeus-Critias, Proceedings of the IV Symposium Platonicum, International Plato Studies, ${ }^{\circ} 9$, Academia Verlag, Sankt Augustin, 1997, p. 281-288. 25 Pour cette interprétation, voir C.STEEL, « The moral purpose of the human body. A reading of Timaeus 69-72”, Phronesis 46, n², 2001, p.105-128.
} 